\title{
Nacionalidade das Empresas e Fluxo de Empregos: Evidências da Indústria Brasileira de Transformação ${ }^{\star}$
}

\author{
- Luiz A. Esteves* - Pedro S. Martins**
}

\begin{abstract}
Resumo
A segunda metade da década de 90 é um período caracterizado por um grande aumento dos investimentos (e desinvestimentos) externos no Brasil. Essa mudança estrutural da economia brasileira é a motivação para este estudo, cujo objetivo é analisar o efeito da nacionalidade das empresas sobre o fluxo de empregos industrial. Trata-se de um tema sobre o qual há pouca evidência, mesmo para países desenvolvidos, embora não seja raro considerar as empresas estrangeiras como mais 'voláteis' que as empresas domésticas. Usando dados da RAIS e de outras bases e uma amostra de empresas domésticas e estrangeiras, comparamos várias medidas de fluxos de empregos nos dois tipos de empresas. Os resultados apresentados neste trabalho sugerem não haver diferenças significativas nos fluxos de empregos das empresas domésticas e estrangeiras.
\end{abstract}

\section{Palavras-Chave}

empresas estrangeiras, destruição de emprego, 'matching'

\begin{abstract}
The late 1990s in Brazil are a period characterized by a large increase in foreign direct investment inflows (and outflows). This process motivates the present study about job flows in domestic and foreign firms. Although foreign firms tend to be considered more 'footloose' than domestic firms, there is very little evidence on this difference, even for developed countries. Using data from RAIS, a large matched employeremployee panel, and other data sets and a sample of foreign and domestic firms, we compare different measures of job flows across the two types of firms. We do not find evidence of job flows' differences between domestic and foreign firms.
\end{abstract}

\section{Keywords}

foreign firms, job destruction, matching

\section{JEL Classification}

$\mathrm{J} 31, \mathrm{~J} 63, \mathrm{~F} 23$

+ Os autores são gratos pelos comentários e sugestões dos pareceristas anônimos. Todos os erros e omissões são de nossa responsabilidade.

* Departamento de Economia, UFPR. Endereço para contato: Universidade Federal do Paraná - Departamento de Economia - Av. Pref. Lothário Meissner, 632 - Jardim Botânico. Curitiba - PR. CEP: 80210170. E-mail: esteves@ufpr.br.

** Queen Mary, Universidade de Londres; CEG-IST Lisboa e IZA. E-mail: p.martins@qmul.ac.uk.

(Recebido em fevereiro de 2008. Aceito para publicação em agosto de 2009). 


\section{Introdução}

A segunda metade da década de 90 é um período de particular importância para a economia brasileira por conta da estabilização econômica (após décadas de alta inflação e diversas reformas monetárias). Além disso, esse período - analisado neste trabalho - também é caracterizado no plano microeconômico por privatizações, fusões e um volume sem precedentes de ingresso de investimentos externos diretos (IED).

Em relação a este último aspecto, o investimento externo, a Figura 1 mostra a evolução do volume de recursos líquidos recebidos como IED na economia brasileira desde 1980 até o ano de 2000. Verifica-se que os fluxos líquidos recebidos pelo Brasil chegam a atingir 7 mil milhões de dólares em 1999, quando não ultrapassavam 2 mil milhões por ano até o princípio da segunda metade da década.

Esta mudança estrutural da economia brasileira é fonte de motivação para o presente estudo, cujo objetivo é analisar o efeito da nacionalidade das empresas sobre o fluxo de empregos industrial. O período aqui analisado, 1995 a 2000, é de grande riqueza para tal finalidade, também porque se verifica durante esses anos uma grande quantidade de empresas trocando de nacionalidade por conta de investimentos e desinvestimentos externos, ou seja, empresas domésticas sendo adquiridas por investidores estrangeiros e vice-versa (KPMG, 2001).

A evolução destas empresas é de particular interesse para qualquer estudo sobre empresas estrangeiras e domésticas, na medida em que é possível lidar de melhor maneira com as diferenças em termos de 'heterogeneidade não observada' entre os dois tipos de empresas. Em outras palavras, quando as mesmas empresas são controladas por diferentes investidores pode ser mais fácil separar o efeito do controle da empresa do efeito das diferentes características dessas mesmas empresas.

Os estudos dos impactos da nacionalidade das empresas sobre o mercado de trabalho doméstico tendem a focar a questão do diferencial de salários. Ampla evidência empírica neste sentido é disponível para os países desenvolvidos (CONYON et al. 2002; MARTINS, 2004; GIRMA; GÖRG 2007; ANDREWS et al. 2007) e países em desenvolvimento/emergentes (AITKEN et al. 1996; FEENSTRA; HANSON, 1997; LIPSEY; SJÖHOLM, 2006; EARLE; TELEGDY, 2007), embora esta literatura não tenha ainda concluído se a diferença salarial entre os dois tipos de empresa é significativa. ${ }^{1}$

1 Estudos recentes, usando dados individuais em painel (MARTINS, op. cit.; HEYMAN et al., 2006; ANDREWS et al., 2007) sugerem que as diferenças obtidas em estudos 'cross-section' se devem a heterogeneidade não observada ou efeitos de seleção. 


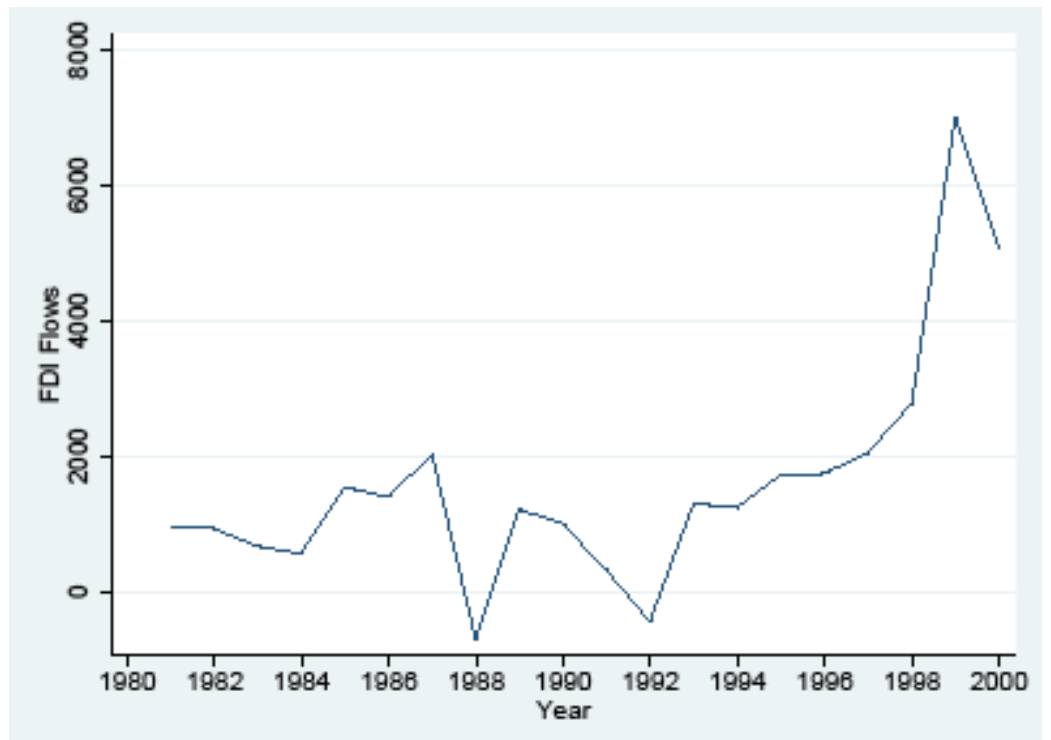

Figura 1 - Investimento Externo Direto no Brasil (Manufatura), 1980-2000

Fonte: Muendler (2003). Unidade: Milhões de dólares

No entanto, é muito limitado o número de análises sobre o efeito da nacionalidade das empresas em termos dos fluxos de empregos. Por outro lado, a literatura sobre fluxos de empregos (DAVIS et al., 1996) tem crescido bastante e já apresenta vários fatos estilizados. Por exemplo, há evidência de elevados níveis de realocação de empregos e de trabalhadores (ibidem), em que a força de trabalho de cada empresa tende a aumentar ou diminuir cerca de $10 \%$ em cada ano. Em geral, estes fluxos também exibem um nível elevado de persistência e ciclicidade. ${ }^{2}$

Por outro lado, os únicos estudos que conhecemos que abordam a eventual maior volatilidade das empresas estrangeiras em termos dos seus níveis de emprego tendem a seguir uma abordagem diferente. Estes trabalhos (GÖRG; STROBL, 2003; BERNARD; SJÖHOLM, 2003; ALVAREZ; GÖRG, 2005) consideram as diferenças entre empresas domésticas e estrangeiras em termos da probabilidade de saírem do mercado, ignorando a dimensão da criação/destruição de empregos. Além disso, a evidência que apresentam é contraditória entre si, sugerindo que os efeitos das empresas estrangeiras dependem do país ou período analisado ou ainda da metodologia adotada.

Este trabalho procura cobrir esta brecha na literatura, fornecendo evidência empírica para o caso das empresas industriais brasileiras. Utilizamos informações

2 Ver Ribeiro et al. (2004) para uma aplicação ao caso brasileiro. 
de três diferentes bases de dados: RAIS (Relação Anual de Informações Sociais do Ministério do Trabalho e Emprego), Censo de Capitais Estrangeiros do Banco Central do Brasil, de 1995, e uma terceira base de dados compilada por DeNegri (2003). ${ }^{3}$

Este artigo é estruturado da seguinte maneira: a seção 2 apresenta brevemente as definições e os fatos estilizados da literatura sobre fluxos de empregos; a seção 3 apresenta as bases de dados, variáveis utilizadas no trabalho e suas respectivas estatísticas descritivas; a seção 4 apresenta o modelo econométrico e os respectivos resultados do efeito da nacionalidade das empresas sobre seus fluxos de emprego; a seção 5 é destinada a contornar o problema de autosseleção da nacionalidade das empresas e desta forma obter diferenciais de fluxos não enviesados; a última seção é destinada às conclusões e considerações finais.

\section{Fluxo de Empregos}

\subsection{Definições}

As últimas décadas têm mostrado um grande esforço da literatura econômica na compreensão da dinâmica dos fluxos de emprego, uma vez que tal análise é de fundamental importância para um melhor entendimento do funcionamento do mercado de trabalho e da realocação de recursos humanos de uma determinada economia sujeita a choques exógenos ou endógenos.

Davis e Haltiwanger (1999) argumentam que nas economias ocidentais desenvolvidas (EUA, Canadá e Europa Ocidental) cerca de um em cada dez postos de trabalho é criado e um em cada dez postos de trabalho é destruído a cada ano. Estes valores conduzem a uma elevada realocação de trabalhadores - o que implica elevadas taxas de rotatividade, fato este que não pode ser capturado através da simples análise de mudança líquida do emprego.

Sabe-se que as taxas de criação, destruição e realocação de empregos observadas nas empresas são, em grande medida, respostas a choques, mudanças estruturais, ciclos econômicos etc. Como já mencionado anteriormente, a segunda metade da década de 90 é caracterizada como um período de mudança estrutural da economia brasileira. Desejamos saber se tais fluxos foram significativamente diferentes para as

3 Esta base de dados reporta as mudanças de nacionalidade das empresas do setor manufatureiro para o período 1996-2000 e será apresentada com maior detalhe nas seções posteriores. 
empresas domésticas e estrangeiras; para tanto, se faz necessário introduzir algumas definições sobre fluxos de empregos.

Todas as variáveis de fluxos são definidas conforme o padrão da literatura (DAVIS et al., 1996). Cada taxa é construída a partir da divisão de um dado fluxo pela média de emprego de cada firma em dois períodos distintos. A taxa de criação de empregos é definida como:

$$
J C_{t}=\frac{L_{t}-L_{t-1}}{0,5\left(L_{t}+L_{t-1}\right)}
$$

se $L_{t}>L_{t-1}$, ou 0 , se $L_{t}<L_{t-1}$, em que $L_{t}$ é o número de trabalhadores no período $t$.

Similarmente, a taxa de destruição de empregos é definida como

$$
J D_{t}=\frac{L_{t-1}-L_{t}}{0,5\left(L_{t}+L_{t-1}\right)}
$$

se $L_{t}<L_{t-1}$, ou 0 , se $L_{t}>L_{t-1}$. Adicionalmente, a taxa de criação líquida de empregos $\left(\mathrm{NJC}_{\mathrm{t}}\right)$ corresponde a $\mathrm{JC}_{\mathrm{t}}-\mathrm{JD}_{\mathrm{t}}$ e a taxa de realocação de empregos $\left(J R_{\mathrm{t}}\right)$ é igual a $\mathrm{JC}_{\mathrm{t}}+\mathrm{JD}_{\mathrm{t}}$.

\subsection{Fatos Estilizados}

Alguns fatos estilizados caracterizam a literatura sobre o tema, tais como a grande realocação existente para que sejam criados novos empregos, o fato de o setor não industrial apresentar taxas de criação de postos de trabalho superiores ao setor industrial e o comportamento dos fluxos de criação e destruição de postos de trabalho em relação ao ciclo econômico.

Diferenças nos fluxos de emprego para empresas com diferentes escalas e graus de maturidade também são alvos de estudos na literatura: as empresas pequenas e mais jovens apresentam maiores taxas de criação e destruição de emprego do que aquelas mais maduras e de maior porte, o que pode ser justificado por processos de ajustamento de escala (escala eficiente) e aprendizado (JOVANOVIC, 1982). 
(DAVIS et al., 1996) apresentam os principais fatos estilizados sobre o tema. ${ }^{4}$ Segundo eles, são quatro fatos principais: 1) Magnitude: as taxas de criação e destruição de postos de trabalho são elevadas: considerando o período de um ano, em média, um em cada dez postos de trabalho é destruído; contudo, um número comparável de postos são criados em outras atividades; 2) Persistência: é verificada uma alta persistência nas taxas de criação e destruição, tendo em vista que a maior parte dos postos destruídos não reabre na mesma localidade em um intervalo de dois anos; 3) Concentração: ocorre uma concentração das taxas de criação e destruição em firmas que apresentam grandes mudanças no emprego, cerca de dois terços das taxas de criação e destruição se devem a empresas que expandem ou retraem 25\% ou mais o seu emprego em um intervalo de 1 ano; 4) Ciclo: as taxas de destruição apresentam uma resposta ao ciclo maior que as taxas de criação. As recessões são caracterizadas por um grande aumento das taxas de destruição e por uma pequena queda na taxa de criação.

Verificamos assim que fatores como a idade da firma, sua escala e suas características tecnológicas, tais como a intensidade de capital, são fundamentais para determinar seus fluxos de emprego. Dada a heterogeneidade entre as empresas domésticas e estrangeiras no Brasil, tais informações sobre os fatos estilizados justificarão algumas das estratégias de identificação a serem utilizadas ao longo deste trabalho.

\section{Dados}

\subsection{Descrição das Bases}

A principal fonte de dados utilizada neste trabalho é a RAIS (Relação Anual de Informações Sociais) do Ministério do Trabalho e Emprego. Os dados provenientes da RAIS fornecem informação censitária anual das empresas e trabalhadores que compõem o setor formal da economia brasileira.

4 Gómez-Salvador, Messina e Vallanti (2004) também avançam na categorização dos principais fatos desta literatura: 1) um grande número de postos de trabalho são criados e destruídos em todos os países e em todos os setores, independentemente da fase do ciclo econômico; 2) as taxas de criação e destruição de empregos são negativamente correlacionadas, mas não de forma perfeita; 3) a criação de empregos é pró-cíclica e a destruição é contracíclica; 4) as volatilidades das duas taxas podem diferir entre os diversos países; 5) a taxa de realocação é inversamente relacionada com a intensidade de capital das empresas, de forma que mais empregos são criados e destruídos no setor de serviços do que na indústria; 6) a intensidade da realocação depende de várias características das firmas como idade e tamanho, que são inversamente relacionadas com a taxa de criação de empregos; e, por fim, 7) a taxa de realocação é um fenômeno persistente, o que faz com que políticas temporárias de emprego surtam pouco efeito, uma vez que existe uma característica estrutural nos fluxos de trabalho. 
Estes dados incluem informações individuais de firmas (tamanho, indústria, localização, número de estabelecimentos etc.) e trabalhadores (escolaridade, gênero, idade, tempo de emprego, horas trabalhadas, remuneração, ocupação etc.). ${ }^{5}$ Além disso, como os registros da RAIS incluem todos os trabalhadores que passaram por uma determinada empresa durante $\mathrm{o} \mathrm{ano}^{6}$, as medidas relacionadas com os trabalhadores (capital humano, por exemplo) são ponderadas por seu tempo de permanência na empresa durante o ano analisado.

Uma segunda fonte de informações utilizada neste trabalho é o Censo de Capitais Estrangeiros do Banco Central do Brasil, referente aos anos de 1995 e 2000. Esta pesquisa fornece informações sobre a composição do capital para todas as empresas brasileiras que dispõem de participação estrangeira. O critério aqui utilizado para designar empresas estrangeiras é a participação acionária superior a 50\% do capital.

Uma vez que o Censo de Capitais Estrangeiros é realizado a cada cinco anos (1995, 2000 e 2005), utilizou-se neste trabalho uma terceira fonte de dados contendo informações relativas às mudanças de nacionalidade das empresas no período 1996-1999. Esta pesquisa foi conduzida por DeNegri (op.cit.) e abrange apenas as empresas industriais do setor manufatureiro. Esta restrição deve-se ao método utilizado pela autora para identificar as mudanças de composição de capital no período: a autora utilizou as informações anuais de mudanças estruturais das empresas junto à PIA (Pesquisa Industrial Anual) do IBGE (Instituto Brasileiro de Geografia e Estatística). Por questões de especificidades da base de dados (conforme mencionado acima), a nossa amostra é restrita às empresas do setor industrial manufatureiro.

A nossa amostra é constituída de aproximadamente 28.000 empresas/ano com tamanho mínimo de 10 empregados $^{7}$, conforme reportado na Tabela 1 a seguir:

5 Ver Martins e Esteves (2006) e Menezes-Filho e Muendler (2007) para exemplos recentes de utilização desta base de dados.

6 Algumas pesquisas interdomésticas similares à RAIS, tais como a portuguesa "Quadros de Pessoal", fornecem apenas informações individuais dos trabalhadores que compõem o estoque de emprego das empresas num determinado dia ou mês de cada ano.

7 Optamos por considerar apenas empresas com dez ou mais trabalhadores, uma vez que há uma grande quantidade de variáveis com "missings" para empresas de menor porte. 
Tabela 1 - Quantidades de Empresas

\begin{tabular}{lccccc}
\hline & \multicolumn{2}{c}{ Empresas $>=10$ empregados } & & \multicolumn{2}{c}{ Empresas $>=100$ empregados } \\
\cline { 2 - 3 } \cline { 5 - 6 } Ano & Domésticas & Estrangeiras & & Domésticas & Estrangeiras \\
\hline 1995 & 27308 & 872 & & 5128 & 600 \\
1996 & 27302 & 878 & & 5035 & 599 \\
1997 & 26916 & 1264 & & 4864 & 857 \\
1998 & 26901 & 1279 & & 4702 & 867 \\
1999 & 26889 & 1291 & & 4571 & 858 \\
2000 & 27005 & 1175 & & 4694 & 805 \\
\hline
\end{tabular}

Quando consideramos apenas as empresas industriais com mais de cem empregados, o número de empresas da amostra cai para aproximadamente 5.500 empresas/ano. $\mathrm{Na}$ Tabela 1, o leitor poderá verificar que as empresas estrangeiras empregam, majoritariamente (aproximadamente 70\%), mais de cem funcionários em suas plantas. Já no caso das empresas domésticas, não mais que 20\% destas empregam mais do que cem funcionários.

\subsection{Estatísticas Descritivas}

As principais variáveis utilizadas ao longo deste trabalho são reportadas, conjuntamente com suas respectivas médias e desvios padrão, nas Tabelas 2 e 3 . Todas as variáveis são computadas ao nível da firma; as variáveis de capital humano, tais como salários, taxa de crescimento dos salários, escolaridade (anos de estudo), tempo de emprego (meses), gênero e experiência (anos) correspondem a valores médios por empresa obtidos a partir de informações individuais dos trabalhadores.

As estatísticas descritivas foram obtidas para todos os anos da amostra (1995-2000), porém reportamos apenas as informações para os anos de $1996^{8}$ e $2000 .^{9}$ Estas descritivas são reportadas por tipo de propriedade (doméstica ou estrangeira) e por diferentes grupos de tamanho: (1) empresas com dez ou mais trabalhadores; e (2) empresas com cem ou mais trabalhadores.

Podemos verificar na Tabela 2 que no ano de 1996 a taxa de criação de empregos (JC) foi superior para as empresas domésticas, enquanto as taxas de destruição (JD) foram maiores para as empresas estrangeiras. Tais resultados são observados

8 Importante lembrar que as informações do ano de 1995 servem apenas de base para os cálculos das taxas de crescimento e fluxos de emprego.

9 As tabelas com os resultados para os demais anos serão encaminhadas aos leitores mediante solicitação. 
para ambas as faixas de tamanho de firma. Observamos também que, sob quaisquer condições, a taxa de realocação de empregos (JR) não é inferior a 0,16 (ou 16\%).

Tabela 2 - Estatísticas Descritivas 1996

\begin{tabular}{|c|c|c|c|c|}
\hline \multirow[b]{2}{*}{ Variáveis } & \multicolumn{2}{|c|}{ Empresas $>=10$ empregados } & \multicolumn{2}{|c|}{ Empresas $>=100$ empregados } \\
\hline & Domésticas & Estrangeiras & Domésticas & Estrangeiras \\
\hline \multirow[t]{2}{*}{$\mathrm{JC}$} & 0,095 & 0,078 & 0,079 & 0,075 \\
\hline & $(0,166)$ & $(0,217)$ & $(0,179)$ & $(0,231)$ \\
\hline \multirow[t]{2}{*}{$J D$} & 0,077 & 0,091 & 0,087 & 0,092 \\
\hline & $(0,138)$ & $(0,144)$ & $(0,138)$ & $(0,131)$ \\
\hline \multirow[t]{2}{*}{ NJC } & 0,018 & $-0,013$ & $-0,008$ & $-0,017$ \\
\hline & $(0,248)$ & $(0,287)$ & $(0,255)$ & $(0,290)$ \\
\hline \multirow[t]{2}{*}{$J R$} & 0,173 & 0,169 & 0,167 & 0,168 \\
\hline & $(0,178)$ & $(0,230)$ & $(0,193)$ & $(0,237)$ \\
\hline \multirow[t]{2}{*}{ In Salário Real } & 6,748 & 7,888 & 7,116 & 7,873 \\
\hline & $(0,604)$ & $(0,491)$ & $(0,576)$ & $(0,487)$ \\
\hline \multirow[t]{2}{*}{ Delta In Salário Real } & $-0,003$ & $-0,038$ & $-0,023$ & $-0,036$ \\
\hline & $(0,416)$ & $(0,379)$ & $(0,428)$ & $(0,417)$ \\
\hline \multirow[t]{2}{*}{ Escolaridade } & 6.309 & 8.498 & 6.418 & 8.381 \\
\hline & $(1,840)$ & $(1,990)$ & $(1,860)$ & $(1,850)$ \\
\hline \multirow[t]{2}{*}{ Experiência } & 18.757 & 18.767 & 19.329 & 18.643 \\
\hline & $(4,740)$ & $(3,510)$ & $(3,770)$ & $(3,240)$ \\
\hline \multirow[t]{2}{*}{ Tempo de Emprego } & 40.189 & 67.471 & 50.407 & 71.322 \\
\hline & $(22,090)$ & $(29,210)$ & $(25,670)$ & $(28,490)$ \\
\hline \multirow[t]{2}{*}{ Idade da Firma } & 16.896 & 26.276 & 25.621 & 29.188 \\
\hline & $(10,640)$ & $(10,460)$ & $(11,920)$ & $(9,960)$ \\
\hline \multirow[t]{2}{*}{ Emprego } & 106,700 & 594,540 & 430,310 & 844,810 \\
\hline & $(398,710)$ & $(1516,340)$ & $(855,400)$ & $(1781,670)$ \\
\hline \multirow[t]{2}{*}{ Proporção Mulheres } & 0,286 & 0,225 & 0,271 & 0,229 \\
\hline & $(0,268)$ & $(0,167)$ & $(0,219)$ & $(0,176)$ \\
\hline Observaç̃es & 27302 & 878 & 5035 & 599 \\
\hline
\end{tabular}

Notas: (1) Desvio padrão entre parênteses; (2) Salários a preços de 2000, deflacionados pelo INPC.

Podemos verificar que as empresas estrangeiras são maiores, mais maduras (idade da firma) e seus trabalhadores apresentam maiores salários, níveis de escolaridade e tempo de emprego. Por outro lado, as empresas domésticas empregam fração maior de mulheres em seus quadros de pessoal.

Mesmo quando restringimos a amostra para empresas com cem trabalhadores ou mais, verificamos que as diferenças entre as empresas domésticas e nacionais permanecem significativas, embora tal corte tenha eliminado mais de 22.200 empresas 
nacionais (aproximadamente $80 \%$ das empresas), enquanto a redução de empresas estrangeiras tenha sido de apenas 279 empresas (32\%).

Em geral, os resultados apresentados para o ano de 1996 são similares àqueles obtidos (e não reportados) para os anos de 1997 a 1999, exceto se for considerado o fato de as taxas de realocação (JR) serem ligeiramente menores (em média 15\%).

Os dados para o ano de 2000 apresentam algumas informações diferentes daquelas observadas para todos os demais períodos: (1) as empresas domésticas apresentam taxas de destruição (JD) não inferiores às taxas apresentadas pelas empresas estrangeiras; (2) pela primeira vez na série histórica é encontrada uma taxa de realocação (JR) inferior a $14 \%$.

Tabela 3 - Estatísticas Descritivas 2000

\begin{tabular}{lcccc}
\hline & \multicolumn{2}{c}{ Todas as empresas } & \multicolumn{2}{c}{ Empresas $>=100$ empregados } \\
Variáveis & Domésticas & Estrangeiras & Domésticas & Estrangeiras \\
\hline JC & 0,078 & 0,077 & 0,097 & 0,083 \\
& $(0,128)$ & $(0,150)$ & $(0,158)$ & $(0,167)$ \\
JD & 0,077 & 0,062 & 0,048 & 0,045 \\
NJC & $(0,167)$ & $(0,162)$ & $(0,132)$ & $(0,122)$ \\
& 0,001 & 0,015 & 0,049 & 0,038 \\
JR & $(0,237)$ & $(0,242)$ & $(0,213)$ & $(0,225)$ \\
& 0,155 & 0,140 & 0,146 & 0,128 \\
In Salário Real & $(0,179)$ & $(0,200)$ & $(0,182)$ & $(0,188)$ \\
& 6,141 & 7,195 & 6,407 & 7,177 \\
Delta In Salário Real & $(0,516)$ & $(0,513)$ & $(0,523)$ & $(0,500)$ \\
& 0,011 & 0,017 & 0,005 & 0,013 \\
Escolaridade & $(0,127)$ & $(0,112)$ & $(0,108)$ & $(0,110)$ \\
& 7.032 & 9.394 & 7.318 & 9.375 \\
Experiência & $(1,760)$ & $(1,810)$ & $(1,780)$ & $(1,720)$ \\
& 19.662 & 18.295 & 18.898 & 17.852 \\
Tempo de Emprego & $(4,820)$ & $(3,400)$ & $(3,880)$ & $(2,980)$ \\
& 52.225 & 73.129 & 55.274 & 73.887 \\
Idade da Firma & $(25,170)$ & $(30,450)$ & $(26,530)$ & $(29,760)$ \\
& 20.801 & 30.103 & 28.096 & 32.907 \\
Emprego & $(10,580)$ & $(10,950)$ & $(12,380)$ & $(10,730)$ \\
& 93,280 & 590,610 & 373,630 & 836,700 \\
Proporção Mulheres & $(330,750)$ & $(1503,450)$ & $(729,430)$ & $(1762,890)$ \\
Observações & 0,286 & 0,222 & 0,269 & 0,222 \\
\hline & $(0,263)$ & $(0,163)$ & $(0,221)$ & $(0,167)$ \\
& 27005 & 1175 & 4694 & 805 \\
\hline
\end{tabular}

Notas: (1) Desvio padrão entre parênteses; (2) Salários a preços de 2000, deflacionados pelo INPC. 


\section{Resultados}

\subsection{Estratégia de Identificação}

Foi mencionado nas seções anteriores que algumas das características das firmas, tais como seu tamanho, idade e intensidade de capital são responsáveis por explicar seus fluxos de empregos, logo, não podemos nos restringir a analisar as diferenças de fluxos entre empresas domésticas e estrangeiras apenas analisando suas taxas médias JC, JD, NJC e JR. Este argumento é reforçado pelo fato de que a heterogeneidade entre estes grupos de empresas permanece mesmo quando restringimos nossa amostra para empresas com maior escala (empresas com cem ou mais trabalhadores).

Esta seção é dedicada a analisar o impacto da nacionalidade das empresas sobre seus respectivos fluxos de emprego, controlando para várias outras possíveis diferenças entre os dois tipos de empresas. Explorando o caráter longitudinal da nossa base de dados é possível utilizar uma especificação econométrica que nos permita o controle da heterogeneidade não observada das firmas. O modelo a ser estimado apresenta a seguinte especificação:

$$
f_{i t}=x_{i t}^{\prime} \alpha+f_{i t}^{\prime} \beta+\text { For }_{i t} \eta+\rho_{i}+\gamma_{t}+\varepsilon_{i t}
$$

onde $f_{i t}$ representa um determinado fluxo (JC, JD, NJC e JR) da firma $i$ no período $t, x^{\prime}$ é um vetor de características dos trabalhadores agregados ao nível da firma (escolaridade, tempo de emprego, experiência e proporção de mulheres), $f^{\prime}$ é um vetor de controles das firmas (idade da firma e emprego), $\eta$ é o parâmetro de interesse, indicando a diferença média nos fluxos entre empresas domésticas (For $=0$ ) e estrangeiras (For $=1$ ). A especificação acima inclui ainda o termo $\rho_{i}$ relativo aos efeitos fixos das firmas, o termo $\gamma_{t}$ relativo às dummies de tempo e $\varepsilon$ é o termo de erro aleatório.

\subsection{Estimativas}

Os resultados das estimativas obtidas para o modelo especificado na equação (1) são reportados na Tabela 4. Cada valor de coeficiente apresentado equivale a uma regressão diferente, ou seja, ao todo foram estimadas oito regressões distintas e, por motivo de limitação de espaço, omitimos a apresentação dos coeficientes estimados para as demais variáveis de controle. ${ }^{10}$

10 As tabelas com todos os resultados serão encaminhadas aos leitores mediante solicitação. 
As regressões foram obtidas para cada fluxo de emprego (variável dependente) distinto (JC, JD, NJC e JR) e para dois grupos amostrais: (1) o primeiro conjunto é formado por empresas com dez ou mais trabalhadores; e (2) o segundo grupo é uma subamostra do grupo anterior, onde consideramos apenas empresas com cem ou mais trabalhadores.

$\mathrm{Na}$ Tabela 4 podemos observar que as empresas estrangeiras apresentam menores taxas de criação de emprego quando comparadas com as empresas domésticas. Este diferencial nas taxas de criação é de $1,3 \%$. No entanto, tal resultado não é observado quando restringimos a amostra para empresas com cem ou mais trabalhadores. Neste segundo caso não é constatada nenhuma diferença significativa entre os dois grupos de empresas.

Quando o foco da análise são as taxas de destruição, pode-se constatar que não há nenhuma evidência de diferenças significativas entre empresas domésticas e estrangeiras. Este resultado é observado para qualquer tipo de amostra selecionada. A mesma conclusão pode ser verificada quando analisamos as diferenças nas taxas de realocação das firmas domésticas e estrangeiras.

Tabela 4 - Regressões de Painel, Efeitos Fixos para Firmas

\begin{tabular}{lcc}
\hline Variável Dependente & $\begin{array}{c}\text { Empresas }>=10 \\
\text { Empregados }\end{array}$ & $\begin{array}{c}\text { Empresas }>=100 \\
\text { Empregados }\end{array}$ \\
\hline JC & $-0,013$ & 0,007 \\
& $(0,005)^{\star *}$ & $(0,007)$ \\
JD & 0,002 & 0,0001 \\
& $(0,005)$ & $(0,007)$ \\
NJC & $-0,015$ & 0,007 \\
& $(0,009)^{\star}$ & $(0,011)$ \\
JR & $-0,010$ & 0,007 \\
& $(0,007)$ & $(0,009)$ \\
Observações & 140765 & 27852 \\
\hline
\end{tabular}

Notas: (1) Nível de significância 0,01 $\left({ }^{* * *}\right), 0,05\left({ }^{* *}\right), 0,10\left(^{*}\right) ;(2)$ Os valores em negrito referem-se aos coeficientes da variável binária explicativa Estrangeira; (3) Cada coeficiente equivale ao resultado de uma regressão; (4) Erros padrão entre parênteses; (5) Cada regressão inclui as seguintes variáveis explicativas (não reportadas na tabela) de controle: escolaridade, experiência, tempo de emprego, proporção mulheres, ln emprego, idade da firma e dummies de ano.

Os resultados obtidos nesta seção sugerem não haver grandes diferenças nos fluxos de empregos das empresas domésticas e estrangeiras - exceto para o caso da taxa de criação de empregos (JC), onde as empresas (com dez trabalhadores ou mais) 
domésticas apresentam taxas de criação 1,3 pontos percentuais superiores àquelas observadas pelas empresas estrangeiras.

Um ponto merece especial atenção e cautela na interpretação de tais resultados. $\mathrm{Na}$ Tabela 1 deste artigo o leitor verificará que o número de empresas estrangeiras da amostra sobe de 872 em 1995 para 1175 empresas em 2000, ou seja, uma elevação em 303 unidades. Esse diferencial corresponde ao saldo de 453 empresas domésticas que se tornaram estrangeiras e 150 empresas estrangeiras que se tornaram domésticas. Algumas questões devem ser levantadas: (i) tais investimentos e desinvestimentos externos foram aleatórios? (ii) há um viés de seleção que caracteriza a nacionalidade das empresas?

Quando propomos cautela ao leitor na interpretação dos resultados da Tabela 4, estamos sugerindo que tais resultados podem estar enviesados caso haja um processo não aleatório que determine a nacionalidade das firmas, uma vez que a nãoaleatoriedade é uma hipótese do modelo econométrico descrito na equação (1). A próxima seção é destinada a tentar solucionar tal problema.

\section{Nacionalidade das Empresas e Viés de Seleção}

\subsection{Propensity Score Matching}

Caso a distribuição entre firmas domésticas e estrangeiras fosse aleatória dentro da amostra, estimar o efeito médio da nacionalidade sobre os fluxos de empregos seria muito simples: bastaria testar ex post a diferença de médias das variáveis supostamente impactadas (JC, JD, NJC e JR) pela nacionalidade das empresas.

Entretanto, tal problema não é trivial, uma vez que as firmas podem se autosselecionarem entre nacionais e estrangeiras - e isso deve ser levado em consideração para responder às perguntas que são endereçadas neste trabalho. Quando os determinantes da autosseleção são conhecidos ou, no caso, se soubermos quais as variáveis que levam uma empresa a ser doméstica ou estrangeira, ou até mesmo mudar de nacionalidade, pode-se recorrer aos chamados experimentos quase-naturais. Basicamente, o que essa metodologia faz é sofisticar o teste de médias dos experimentos naturais a fim de comparar firmas com características semelhantes.

Dentro desse arcabouço metodológico, a técnica escolhida no presente trabalho é o propensity score matching (PSM). O PSM permite um controle do viés de autosseleção a partir da restrição da amostra original a duas amostras comparáveis, de 
forma que os grupos de casos e controles tenham características tão semelhantes a ponto de se supor que, entre essas duas amostras comparáveis, a distribuição do "tratamento" (o fato de a firma estrear ou não) seja pseudoaleatória.

O PSM é um procedimento de dois estágios. No primeiro estágio estima-se a probabilidade das firmas serem estrangeiras. Depois, faz-se o emparelhamento das probabilidades estimadas. O emparelhamento é realizado da seguinte forma: seja $\hat{p}_{i}\left(X_{i}\right)$ a probabilidade de a firma $i$ ser estrangeira, de maneira que $i$ efetivamente seja estrangeira. Se, dentro de um raio (pequeno) partindo de $\hat{p}_{i}\left(X_{i}\right)$, existir pelo menos um $\hat{p}_{j}\left(X_{j}\right)$, sendo $j$ uma firma doméstica, as firmas $i$ e $j$ formarão um par caso-controle, respectivamente. Então, esses grupos são comparados a fim de mensurar o efeito da nacionalidade sobre as variáveis de interesse (JC, JD, NJC e JR).

O emparelhamento é feito a partir do algoritmo greedy no software SAS. Esse algoritmo casa pares de firmas de acordo com uma precisão especificada. Por exemplo, um matching a dois dígitos significa que uma firma estreante com $\hat{p}(X)=0,5674$ será casada com uma firma não estreante com $\hat{p}(X)=0,56 x p t o$, em que o xpto indica quaisquer algarismos porque os dois primeiros algarismos já são iguais. Assim, no caso do matching a dois dígitos, a diferença entre as probabilidades casadas não é superior a $1 \%$. Em um matching a três dígitos, essa mesma firma seria casada com uma não exportadora com $\hat{p}(X)=0,567$ pto, de forma que esse emparelhamento é mais preciso do que o anterior (a diferença entre as probabilidades não é maior do que $0,1 \%)$, e assim por diante.

O emparelhamento foi feito para cada ano da amostra, e as quantidades de empresas nacionais e estrangeiras pareadas são reportadas na Tabela 5:

\section{Tabela 5 - Pareamento do Propensity Score Matching}

\begin{tabular}{lcc}
\hline Ano & Domésticas & Estrangeiras \\
\hline 1996 & 645 & 645 \\
1997 & 895 & 895 \\
1998 & 876 & 876 \\
1999 & 876 & 876 \\
2000 & 783 & 783 \\
\hline
\end{tabular}

O modelo utilizado para a obtenção das probabilidades estimadas considerou como variáveis de controle aquelas reportadas na Tabela A ao final do artigo. As Tabelas A e B reportam as médias das variáveis de controle para as empresas domésticas e 
estrangeiras pareadas no PSM, bem como suas diferenças, para os anos de 1996 e $2000,{ }^{11}$ respectivamente.

A ausência de diferenças significativas entre as médias das variáveis explicativas para os grupos de tratamento (estrangeiras) e controle (domésticas) sugere que o PSM foi satisfatório pelo fato de parear e agrupar empresas homogêneas. A única variável a apresentar diferença significativa (ao nível de significância de 10\%) entre grupos de tratamento e controle foi o ln do Emprego - exclusivamente para o ano de 1996. Em todos os demais anos, todas as variáveis apresentam médias não significativamente diferentes.

\subsection{Resultados}

$\mathrm{Na}$ Tabela 6, encontramos as médias para as variáveis de fluxos de emprego por tipo de empresa e ano, bem como as diferenças de médias e seus respectivos níveis de significância estatística. No que diz respeito à taxa de criação de empregos, verificamos que diferenças significativas são encontradas apenas para os anos de 1996 e 1998. Em ambos os casos as empresas estrangeiras apresentam médias superiores às das empresas domésticas: para o ano de 1996, esta diferença é de 2,2 pontos percentuais e em 1998 esta diferença é de 1,2. Este resultado, mesmo que não robusto para todos os anos da amostra, contradiz nossos resultados obtidos na análise de regressão (seção 4).

Quando o foco de análise são as taxas de destruição de empregos, a única diferença significativa de médias é verificada no ano de 1999, quando as empresas estrangeiras destruíram empregos a uma taxa de um ponto percentual superior ao das empresas domésticas. Resultado similar é verificado para as taxas de realocação de empregos, em que apenas para o ano de 1998 há diferenças significativas de médias: as empresas estrangeiras realocaram empregos a uma taxa de 1,2 pontos percentuais superiores ao das empresas domésticas.

Dada a pequena quantidade de médias significativamente diferentes entre os grupos de tratamento e controle e a não consistência destas diferenças por fluxos para os diferentes anos da amostra, pode-se concluir, mais uma vez, que os resultados apresentados ao longo deste trabalho não constituem evidência suficiente para inferir sobre nenhum efeito da nacionalidade das empresas sobre seus fluxos de empregos.

11 As tabelas para todos os anos serão encaminhadas aos leitores mediante solicitação. 
Tabela 6 - Teste $\boldsymbol{t}$ de Diferença de Médias de Fluxos de Empregos PSM

\begin{tabular}{|c|c|c|c|}
\hline Variáveis & Domésticas & Estrangeiras & Diferença \\
\hline \multirow[t]{2}{*}{ JC 1996} & 0,055 & 0,077 & $-0,022$ \\
\hline & $(0,005)$ & $(0,008)$ & $(0,009)^{\star \star}$ \\
\hline \multirow[t]{2}{*}{ JD 1996} & 0,106 & 0,094 & 0,011 \\
\hline & $(0,007)$ & $(0,006)$ & $(0,009)$ \\
\hline \multirow[t]{2}{*}{ NJC 1996} & $-0,051$ & $-0,016$ & $-0,034$ \\
\hline & $(0,010)$ & $(0,011)$ & $(0,015)^{\star *}$ \\
\hline \multirow[t]{2}{*}{ JR 1996} & 0,161 & 0,172 & $-0,010$ \\
\hline & $(0,007)$ & $(0,009)$ & $(0,011)$ \\
\hline \multirow[t]{2}{*}{ JC 1997} & 0,077 & 0,074 & 0,003 \\
\hline & $(0,005)$ & $(0,005)$ & $(0,007)$ \\
\hline \multirow[t]{2}{*}{ JD 1997} & 0,069 & 0,076 & $-0,007$ \\
\hline & $(0,004)$ & $(0,004)$ & $(0,006)$ \\
\hline \multirow[t]{2}{*}{ NJC 1997} & 0,007 & $-0,001$ & 0,008 \\
\hline & $(0,007)$ & $(0,007)$ & $(0,011)$ \\
\hline \multirow[t]{2}{*}{ JR 1997} & 0,146 & 0,151 & $-0,005$ \\
\hline & $(0,006)$ & $(0,006)$ & $(0,008)$ \\
\hline \multirow[t]{2}{*}{ JC 1998} & 0,048 & 0,061 & $-0,012$ \\
\hline & $(0,004)$ & $(0,005)$ & $(0,006)^{\star *}$ \\
\hline \multirow[t]{2}{*}{ JD 1998} & 0,097 & 0,096 & 0,001 \\
\hline & $(0,005)$ & $(0,005)$ & $(0,007)$ \\
\hline \multirow[t]{2}{*}{ NJC 1998} & $-0,048$ & $-0,035$ & $-0,013$ \\
\hline & $(0,007)$ & $(0,008)$ & $(0,011)$ \\
\hline \multirow[t]{2}{*}{ JR 1998} & 0,145 & 0,157 & $-0,012$ \\
\hline & $(0,005)$ & $(0,006)$ & $(0,008)^{*}$ \\
\hline \multirow[t]{2}{*}{ JC 1999} & 0,047 & 0,042 & 0,005 \\
\hline & $(0,004)$ & $(0,003)$ & $(0,005)$ \\
\hline \multirow[t]{2}{*}{ JD 1999} & 0,089 & 0,099 & $-0,010$ \\
\hline & $(0,004)$ & $(0,005)$ & $(0,007)^{\star}$ \\
\hline \multirow[t]{2}{*}{ NJC 1999} & $-0,041$ & $-0,057$ & 0,016 \\
\hline & $(0,007)$ & $(0,006)$ & $(0,010)^{*}$ \\
\hline \multirow[t]{2}{*}{ JR 1999} & 0,137 & 0,141 & $-0,004$ \\
\hline & $(0,005)$ & $(0,005)$ & $(0,008)$ \\
\hline \multirow[t]{2}{*}{ JC 2000} & 0,074 & 0,079 & $-0,005$ \\
\hline & $(0,005)$ & $(0,005)$ & $(0,007)$ \\
\hline \multirow[t]{2}{*}{ JD 2000} & 0,072 & 0,069 & 0,003 \\
\hline & $(0,006)$ & $(0,006)$ & $(0,009)$ \\
\hline \multirow[t]{2}{*}{ NJC 2000} & 0,002 & 0,009 & $-0,007$ \\
\hline & $(0,009)$ & $(0,009)$ & $(0,012)$ \\
\hline \multirow[t]{2}{*}{ JR 2000} & 0,146 & 0,149 & $-0,002$ \\
\hline & $(0,007)$ & $(0,007)$ & $(0,010)$ \\
\hline
\end{tabular}

Notas: (1) Nível de significância 0,01 $\left({ }^{* *}\right), 0,05\left({ }^{* *}\right), 0,10\left(^{*}\right)$; (2) Erros padrão entre parênteses. 


\section{Conclusões}

O grande volume de investimento externo direto e de operações de privatização, aquisições e fusões na economia brasileira durante a segunda metade da década de 90 torna este período de particular importância para o estudo dos impactos das empresas estrangeiras sobre o mercado de trabalho doméstico. Com efeito, o mercado de trabalho é uma das dimensões mais importantes de uma economia para avaliar as consequências da globalização em termos do bem-estar dos países (OECD, 2007).

No entanto, verifica-se que pouca atenção é dada à análise do efeito das empresas estrangeiras sobre os fluxos de empregos, sobretudo quando se considera o efeito da mudança de nacionalidade das firmas sobre tais fluxos. Além disso, a limitada evidência empírica disponível é não conclusiva. Por outro lado, alguns analistas tendem a sugerir que as empresas estrangeiras são caracterizadas por maior 'volatilidade' que as empresas domésticas, algo que teria custos em termos do bem-estar dos países que recebem investimentos externos. Este trabalho procura esclarecer esta questão usando informação detalhada para o caso do Brasil.

Ao longo deste trabalho foram utilizadas duas estratégias de identificação com o objetivo de capturar o efeito da nacionalidade das firmas sobre seus fluxos de empregos. Em um primeiro momento, exploramos a característica longitudinal da nossa amostra ao utilizar uma estimativa com efeitos fixos para firmas, de modo a controlar a heterogeneidade não observável destas empresas. Em um segundo momento, a hipótese de não-aleatoriedade da distribuição entre firmas domésticas e estrangeiras é assumida e o método de PSM é utilizado para contornar eventuais problemas de seleção. Em ambos os casos os resultados apresentados não constituem evidência suficiente para inferir sobre nenhum efeito significativo da nacionalidade das empresas sobre seus fluxos de empregos.

\section{Referências}

AITKEN, B.; HARRISON, A.; LIPSEY, R. Wages and foreign ownership: A comparative study of Mexico, Venezuela, and the United States. Journal of International Economics, 40 (3-4), p. 345-371, 1996.

ALVAREZ, R.; GÖRG, H. Multinationals and plant exit: evidence from Chile. IZA, 2005. (Discussion Paper 785 1611).

ANDREWS, M.; BELLMAN, L.; SCHANK, T.; UPWARD, R. The takeover and selection effects of foreign ownership in Germany: an analysis using linked workerfirm data. GEP, University of Nottingham, 2007. (Research Paper 2007/08).

BERNARD, A. B.; SJÖHOLM, F. Foreign owners and plant survival. NBER Working Paper 10039, 2003. 
CONYON, M.; GIRMA, S.; THOMPSOM, S.; WRIGHT, P. The productivity and wage effects of foreign acquisition in the United Kingdom. Journal of Industrial Economics, v. 50, n.1, p. 85-107, 2002.

DAVIS, S.; HALTIWANGER, J.; SCHUH, S. Job Creation and Destruction. Cambridge, MA: MIT Press, 1996.

DAVIS, S. J.; HALTIWANGER, J. Gross job flows. In: ASHENFELTER, O.; CARD, D. (Eds.). Handbook of labor economics. Amsterdan: Elsevier, 3B, 1999.

DAVIS, S. J.; FABERMAN, R. J.; HALTIWANGER, J. The flow approach to labor markets: new data sources and micro-macro links. Journal of Economic Perspectives, v. 20, n. 3, p.326, 2006.

DeNEGRI, F. Desempenho comercial das empresas estrangeiras no Brasil na década de 90. Universidade Estadual de Campinas, 2003. Mimeo.

EARLE, J.; TELEGDY, A. Ownership and wages: Estimating public-private and foreign-domestic differentials using LEED from Hungary, 1986-2003. NBER Working Paper 12997, 2007.

FEENSTRA, R. C.; HANSON, G. H. Foreign direct investment and relative wages: Evidence from mexico's maquiladoras. Journal of International Economics, v. 42, n. 3-4, p. 371-393, 1997.

GIRMA, S.; GÖRG, H. Evaluating the causal effects of foreign acquisition on domestic skilled and unskilled wages. Journal of International Economics, v. 72, n.1, p. 97-112, 2007.

GÓMEZ-SALVADOR, R.; MESSINA, J.; VALLANTI, G. Gross Job Flows and Institutions in Europe, Labour Economics, v. 11, n. 4, p. 469-485, 2004.

GÖRG, H.; STROBL, E. Footloose' multinationals? Manchester School, v. 71, n. 1, p. 1-19, 2003.

HEYMAN, F.; SJÖHOLM, F.; TINGVALL, P. Is there really a foreign ownership wage premium? Evidence from matched employer-employee data. European Institute of Japanese Studies, 2006. (Working Paper 230).

JOVANOVIC, B. Selection and the evolution of industry. Econometrica, v.5 0, n. 3 , p. 649-670, 1982.

KPMG. Mergers and acquisitions in Brazil: an analysis of the 1990s. KPMG Corporate Finance, São Paulo, 2001.

LIPSEY, R.; SJÖHOLM, F. Foreign firms and Indonesian manufacturing wages: An analysis with panel data. Economic Development and Cultural Change, v. 55, n. 1, p. 201-221, 2006.

MARTINS, P. S. Do foreign firms really pay higher wages? Evidence from different estimators. IZA, 2005. (Discussion Paper 1388).

.; ESTEVES, L. A. Is there rent sharing in developing countries? Matchedpanel evidence from Brazil. IZA, 2006. (Discussion Paper 2317). 
MENEZES-FILHO, N.; MUENDLER, M.-A. Labor reallocation in response to trade reform. San Diego: University of California, 2007. Mimeo.

MUENDLER, M.-A. Foreign direct investment by sector of industry, Brazil 19802000. San Diego: University of California, 2003. Mimeo.

OECD. OECD employment outlook. Paris, 2007.

RIBEIRO, E. et al. Trade liberalization, the exchange rate and job flows in Brazil. Journal of Policy Reform, v. 7, n. 4, p. 209-223, 2004.

ROSENBAUM, P.; RUBIN, D.The central role of the propensity score in observational studies for causal effects. Biometrika, v. 70, n. 1, p. 41-55, 1983.

\section{Anexo A - Tabelas Auxiliares}

Tabela A1 - Teste $t$ de Diferença de Médias para Variáveis de Controle do PSM, 1996

\begin{tabular}{lccr}
\hline Variáveis & Domésticas & Estrangeiras & Diferença \\
\hline In Salário Real & 7,743 & 7,759 & $-0,016$ \\
& $(0,017)$ & $(0,018)$ & $(0,026)$ \\
Delta In Salário Real & $-0,028$ & $-0,028$ & 0,000 \\
& $(0,007)$ & $(0,017)$ & $(0,018)$ \\
Tempo de Emprego & 62,912 & 63,618 & $-0,706$ \\
& $(1,085)$ & $(1,137)$ & $(1,572)$ \\
Escolaridade & 8,027 & 8,110 & $-0,082$ \\
& $(0,075)$ & $(0,076)$ & $(0,107)$ \\
Experiência & 18,941 & 18,963 & $-0,021$ \\
& $(0,142)$ & $(0,145)$ & $(0,203)$ \\
Idade da Firma & 25,861 & 25,196 & 0,664 \\
& $(0,447)$ & $(0,418)$ & $(0,612)$ \\
Proporção Mulheres & 0,234 & 0,237 & $-0,003$ \\
& $(0,007)$ & $(0,007)$ & $(0,010)$ \\
In Emprego & 5,25 & 5,14 & 0,109 \\
& $(0,052)$ & $(0,047)$ & $(0,070)^{\star}$ \\
\hline
\end{tabular}

Notas: (1) Nível de significância 0,01 $\left({ }^{* * *}\right), 0,05\left({ }^{* *}\right), 0,10\left({ }^{*}\right) ;(2)$ Erros padrão entre parênteses; (3) Salários a preços de 2000, deflacionados pelo INPC. 
Tabela A2 - Teste $t$ de Diferença de Médias para Variáveis de Controle do PSM, 2000

\begin{tabular}{lrrr}
\hline Variáveis & Domésticas & Estrangeiras & Diferença \\
\hline In Salário Real & 7,021 & 7,019 & 0,002 \\
& $(0,016)$ & $(0,017)$ & $(0,024)$ \\
Delta In Salário Real & 0,014 & 0,016 & $-0,002$ \\
& $(0,004)$ & $(0,004)$ & $(0,006)$ \\
Tempo de Emprego & 66,916 & 68,104 & $-1,188$ \\
& $(1,050)$ & $(1,040)$ & $(1,478)$ \\
Escolaridade & 8,893 & 8,913 & $-0,019$ \\
& $(0,064)$ & $(0,064)$ & $(0,091)$ \\
Experiência & 18,578 & 18,563 & 0,024 \\
& $(0,132)$ & $(0,131)$ & $(0,186)$ \\
Idade da Firma & 27,984 & 28,304 & $-0,319$ \\
& $(0,406)$ & $(0,392)$ & $(0,564)$ \\
Proporção Mulheres & 0,244 & 0,237 & 0,006 \\
In Emprego & $(0,006)$ & $(0,006)$ & $(0,009)$ \\
& 5,062 & 5,072 & $-0,010$ \\
\hline
\end{tabular}

Notas: (1) Nível de significância 0,01 $\left(^{* * *}\right), 0,05\left({ }^{* *}\right), 0,10\left(^{*}\right)$; (2) Erros padrão entre parênteses; (3) Salários a preços de 2000, deflacionados pelo INPC. 